\title{
RNA Interference of the Hemonchus contortus Astacin-like MTP Gene by the Soaking Method
}

\author{
Charles Muleke Inyagwa ${ }^{1 *}$ and Dave $\operatorname{Knox}^{2}$
}

${ }^{1}$ Faculty of Veterinary Medicine \& Surgery, Department of Veterinary Pathology, Microbiology \& Parasitology; Egerton University, P.O. Box 536-20115, Egerton, Kenya

${ }^{2}$ Moredun Research Institute, Pentlands Science Park, Bush Loan, Penicuik, EH26 OPZ, UK

*Corresponding author

\section{A B S T R A C T}

\begin{tabular}{|l|} 
K e y w o r d s \\
RNA interference, \\
$\begin{array}{l}\text { H. contortus, } \\
\text { Astacin-like MTP } \\
\text { gene, Soaking } \\
\text { method }\end{array}$ \\
\hline Article Info \\
$\begin{array}{l}\text { Accepted: } \\
10 \text { March } 2020 \\
\text { Available Online: } \\
10 \text { April } 2020\end{array}$ \\
\hline
\end{tabular}

RNA interference (RNAi) is a powerful molecular tool for gene discovery and functional validation for candidate vaccine antigens from the vast amount of information available within EST datasets. In this paper we report the knock down in the phenotypic expression of Hemonchus contortus astacin-like MTP gene associated with reproduction and bloodfeeding of this parasite which is predominantly expressed in late larval and adult stages by RNAi interference. The RNAi experiments were conducted utilizing the soaking protocol. Results showed that there was interference in expression of each $H$. contortus astacin 3' or 5'ends transcripts individually as judged by RT-PCR's. The resultant dsRNA products were also found to be gene-specific. However no visible phenotypes were detected after simultaneous RNAi treatments for both $H$. contortus astacin 3'and 5'end gene transcripts.

\section{Introduction}

Gut expressed antigens of the parasitic nematode Haemonchus contortus are effective targets for vaccine development (Redmond et al., 2004; Loukas et al., 2004). These proteins play an important role in food digestion within the worm (Williamson et al., 2003) and antibody is thought to mediate the protective immunity after vaccination by blocking the activity of the enzymes. This would interfere with food digestion and may ultimately lead to worm starvation. However, the assumption that intestinal secreted enzymes are important for food digestion is only based on circumstantial evidence. The enzymes are localised in the intestinal villi and are able to degrade proteins involved in nutrition of the worms, such as haemoglobin (Williamson et al., 2003). Nevertheless no 
conclusive experimental evidence to support a specific role in feeding is available. Subjecting specific gut expressed enzymes to RNAi is the way forward to verification of their functions.

A family of astacin-like MTPs associated with the transition to parasitism which are developmentally regulated exist in parasitic nematodes (Zhan et al., 2002; Gomez Gallego et al., 2005). Suggested roles for the MTPs include moulting, feeding, tissue penetration and immunomodulation, thus making them prime targets for intervention by vaccination to control infection.

Astacin-like MTP activity was reported in $H$. contortus L3 EF and in ExL3 ES (Gamble et al., 1989; Geldolf et al., 2005). As with many of the hookworm vaccine candidates, levels of protection using a bacterially-expressed AcMTP have been highly variable.

Assessing astacin-like MTPs functions in RNAi experimental models would provide an insight to their true role in nematodes. This paper describes RNA i of astacin-like MTP in $H$. contortus using the soaking method.

\section{Materials and Methods}

\section{Extraction of RNA from H.contortus parasites}

Total RNA was extracted from exsheathed L3, L4 and adult male and female parasites as described (Hashmi et al., 2002). In brief, parasites were re-suspended in $200 \mu$ l of lysis buffer $(0.5 \%$ SDS, $5 \% \beta$-mercaptoethanol, 10

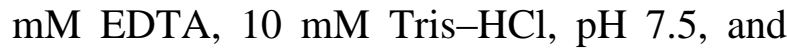
$0.5 \mathrm{mg} / \mathrm{ml}$ proteinase $\mathrm{K}$ ), quick frozen at -80 ${ }^{0} \mathrm{C}$ for $10 \mathrm{~min}$, followed by incubation at 55 ${ }^{0} \mathrm{C}$ for $1 \mathrm{~h}$. The RNA was extracted using Total RNA Isolation Reagent, (Advanced Biotechnologies Ltd.) and stored at $-80^{\circ} \mathrm{C}$ until use.

\section{Generation of $\boldsymbol{H}$. contortus astacin cDNA}

\section{Primers}

Four gene specific primers designated HCF1Nco- I (5'-ccatgga ATGAGGCTCAC TATACTACTG-3'); HCR1Xho- I (5'ctcgaga GGCAGCAAGTTAA

CCAGAT -3'); HCF2-Nco- I (5'-ccatggaAA CTCGGCCACGCTCTTGG-3'); HCR2XhoI (5'-ctcgag GGTGCTTCTCATA ACAA GAAA -3') respectively, based on H.contortus astacin-like MTPs gene (Acc. No.AM159505) (Fig. 1), were synthesized (EurofinS MWG Operon, Germany).

\section{Single step RT-PCR}

Reverse transcriptase coupled PCR (RT-PCR) was used to determine the specificity of $H$. contortus astacin cDNA mRNA transcription using SuperScript One-Step RT-PCR System (Invitrogen) according to the manufacturer's instructions. The thermo cycle conditions were cDNA synthesis and pre-denaturation phase of $55^{\circ} \mathrm{C}$ (30min) (1-cycle) and $94^{\circ} \mathrm{C}$ (5min) (1-cycle), followed by PCR amplification of 30 cycles at $94^{\circ} \mathrm{C}(30 \mathrm{~s}), 56^{\circ} \mathrm{C}$ (1min), $72^{\circ} \mathrm{C}(2 \mathrm{~min})$, and a final extension for $7 \mathrm{~min}$ at $72^{\circ} \mathrm{C}$ in the last cycle.

H. contortus astacin 3'or 5'ends amplified products were analyzed by gel electrophoresis on $1 \%(\mathrm{w} / \mathrm{v})$ agarose gels (Sigma*), stained with red gel (BIOTIUM*) and submerged in 1xTAE electrophoresis buffer. The gels were visualized under ultraviolet illumination and recorded on a Polaroid 667-film. Amplified 3 'and 5'ends of $H$. contortus astacin DNA fragments were excised from agarose gels and purified with QIAqiuck Gel extraction Kit(QIAGEN*), as per manufacturer instructions, and cloned into $\mathrm{pGEM}^{*}-\mathrm{T}$ cloning vector. 
Cloning of $H$. contortus astacin 3 'and 5'ends

The pGEM $^{*}$-T cloning vector (Promega*), and each of the $3^{\prime}$ or 5 'end fragments of purified $H$. contortus astacin DNA obtained from step 1.4.2 above for sub-cloning were combined in approximately 3:1 molar ratio (fragment: vector). The cloning reactions were assembled as follows:

\begin{tabular}{|c|c|c|c|c|}
\hline Reagents & 3'ends & 5 'ends & $+\mathrm{Ve}$ & BG \\
\hline $2 \times$ rapid ligation buffer $T_{4}$ DNA ligase & $5 \mu 1$ & $5 \mu 1$ & $5 \mu 1$ & $5 \mu 1$ \\
\hline pGEM - T (50ng) & $1 \mu 1$ & $1 \mu 1$ & $1 \mu 1$ & $1 \mu \mathrm{l}$ \\
\hline Purified PCR products & $3 \mu 1$ & 3 & - & - \\
\hline Control DNA insert & - & - & $2 \mu 1$ & - \\
\hline$T_{4}$ DNA ligase (3Weiss units $\left./ \mu \mathrm{l}\right)$ & $1 \mu 1$ & $1 \mu 1$ & $1 \mu 1$ & $1 \mu 1$ \\
\hline $\mathrm{dH}_{2} \mathrm{O}$ & - & - & $1 \mu \mathrm{l}$ & $3 \mu \mathrm{l}$ \\
\hline Total volume & $10 \mu \mathrm{l}$ & $10 \mu \mathrm{l}$ & $10 \mu \mathrm{l}$ & $10 \mu 1$ \\
\hline
\end{tabular}

The ligation reactions were incubated overnight at $4{ }^{\circ} \mathrm{C}$. E. coli JM 109 competent cells (STRATAGENE*) were transformed with recombinant $C$. pGEM* ${ }^{*}$-T/ $H$. contortus astacin 3'or 5'end DNA products, respectively.

Transformation of $E$. coli JM 109 with recombinant 3'or 5'ends

The E. coli JM 109 bacteria strain was transformed with recombinant $C$. pGEM* ${ }^{*}$-T/ H. contortus astacin $3^{\prime}$ or 5'ends DNA as per manufacturer instructions (STRATAGENE*). Briefly; $100 \mu 1$ of thawed JM 109 competent cells were mixed with $0.8 \mu \mathrm{l} \quad \beta$ mercaptoethanol $(1.42 \mathrm{M})$ and placed on ice for 10min. A $2 \mu 1$ of ligation mix (recombinant C. pGEM* $-\mathrm{T} / H$. contortus astacin 3'or 5 'ends DNA) was added and the mixture put on ice for 30 minutes.

This was heated at $42^{\circ} \mathrm{C}$ for exactly 45 seconds, and rapidly placed on ice bath for another $2 \mathrm{~min}$. The cells were then cultured in $900 \mu 1$ of SOC media on a slow rotating shaker set at $150 \mathrm{rpm}$ for $90 \mathrm{~min}$ at $37^{\circ} \mathrm{C}$. Transformed cells $(200 \mu \mathrm{l})$ were plated on $1 \%$ (w/v) agarose LB-agar/X-gal $(50 \mathrm{mg} / \mathrm{ml}) / 1 \mathrm{M}$ -
IPTG plates supplemented with $25 \mathrm{mg} / \mathrm{ml}$ of ampicillin antibiotic and incubated at $37^{\circ} \mathrm{C}$ for 16hrs. Largest single colonies were randomly selected and cultured in LB (Luria Bertani medium), (Tryptone (10g), Yeast extract $(5 \mathrm{~g})$, and $\mathrm{NaCl}(10 \mathrm{~g})$ growth media pre-mixed with ampicillin $(25 \mathrm{mg} / \mathrm{ml})$ and incubated overnight at $37^{\circ} \mathrm{C}$ on a slow rotating shaker. The $3^{\prime}$ or 5 'ends plasmid DNA were extracted utilizing Wizard* Plus SV Minipreps DNA purification kit as per manufacturer instructions (PROMEGA*) and stored at $20^{\circ} \mathrm{C}$ until use.

\section{Identification of recombinant 3'or 5'ends}

Recombinant C. pGEM* $-\mathrm{T} / H$. contortus astacin 3'or 5'ends plasmid DNA were digested with Xho I and Nco I restriction enzymes followed by gel electrophoresis for identification purposes and verified by sequencing (EurofinS MWG Operon, Germany).

\section{Construction of recombinant L4440 RNA $A_{i}$ expression vector}

Purified recombinant 3'or 5'ends DNA products obtained from step 1.5.1 above were 
subsequently excised from pGEM $^{*}$-T using Nco I and Xho I restriction enzymes and ligated in the L4440 RNAi vector (Promega*), previously linearized with similar enzymes for construction of RNAi recombinant expression vector (Fig 2). E. coli cells were transformed with recombinant L4440/H. contortus astacin 3'or 5'ends DNA product.

Transformation of $E$. coli with $\mathrm{L4440/H}$. contortus astacin 3 'or 5 'ends

The E. coli XL-10 GOLD* bacteria strain (STRATAGENE*), were transformed with recombinant L4440/H. contortus astacin 3'or 5 'ends DNA essentially as described in step 1.5.1 above and stored at $-20^{\circ} \mathrm{C}$ until use.

\section{Identification of recombinant positive clones}

The recombinant L4440/H. contortus astacin 3'or 5'ends plasmid DNA were digested with Xho I and Nco I restriction enzymes, separated by agar gel electrophoresis for identification and verified by sequencing (EurofinS MWG Operon, Germany).

RNA interference of $\boldsymbol{H}$. contortus astacin gene

\section{Synthesis of dsRNA}

The L4440 constructs were linearised with Nco I and Xho I restriction enzymes separately and dsRNA prepared from these templates using the T7 Ribomax Express RNAi System (Promega*) according to manufacturer instructions.

\section{Soaking of $\boldsymbol{H}$. contortus worms with dsRNA}

RNAi using the soaking protocol was performed on $\sim 100 \mathrm{~L}_{3}$ and $\mathrm{L}_{4}$ worms. The worms were washed with PBS and soaked in
$2 \%$ sodium hypochlorite for $15 \mathrm{~min}$. and washed again.

The parasites in a $30 \mu \mathrm{l}$ of PBS volume containing $1 \mathrm{mg} / \mathrm{ml}$ dsRNA pre-mixed with 3 $\mu \mathrm{l}$ of lipofectin reagent (Invitrogen) were stood at room temperature for $48 \mathrm{~h}$. Larvae soaked in either PBS alone or lipofectin reagent without dsRNA served as the respective controls.

\section{Detection of dsRNA treated worms}

The loss of 3'or 5'end transcripts following RNAi was detected by RT-PCR with pairs of $H$. contortus astacin $3^{\prime}$ or $5^{\prime}$ ends specific primers viz: HCF1Nco- I (5'- ATGAGGCT CACTATACTACTG-3'); HCR1Xho- I (5'GGCAGCAAGTTAA CCAGAT-3'); HCF2Nco-(5'-AACTCGGCCACGCTCTTGG-3'); HCR2Xho- I (5'-GGTGCTTCTCATAACA AGAAA -3'), respectively.

Extraction of t-RNA and the RT-PCR's were performed as previously described. After 35 cycles of amplification, the RT-PCR products were separated on $1 \%$ agarose gels.

\section{Results and Discussion}

\section{H. contortus astacin 3'or 5'end PCR products}

H. contortus astacin 3'or 5'ends PCR products migrated to the expected size of $\sim 200 \mathrm{bp}$ (Fig. 2.0 ), following gel electrophoresis on $1 \%$ (w/v) agarose (Sigma) gels, stained with $0.5 \mathrm{mg} / \mathrm{ml}$ red gel.

\section{Recombinant pGEM -T/ H. contortus astacin 3 'or 5 'ends}

The 3'or 5'ends DNA fragments of $H$. contortus astacin migrated to an expected size of $200 \mathrm{bp}$ (Fig. 3.), after digesting recombinant pGEM $-\mathrm{T} / H$. contortus astacin 
3'or 5'ends with Xho I and Nco I restriction enzymes followed by gel electrophoresis.

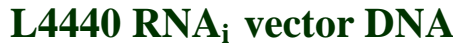

Digested L4440 RNAi vector DNA of $~ 2790$ (Fig. 4), was excised from agarose gels, purified and ligated to purified $H$. contortus astacin 3'or 5'end $s$ fragments of $\sim 200 \mathrm{bp}$ for construction of RNA interference recombinant expression vector of $H$. contortus astacin.

\section{Recombinant $\mathrm{L} 4440 / \mathrm{H}$. contortus astacin 3'or 5'ends}

The recombinant L4440/H. contortus astacin 3'or 5'ends plasmid DNA digested with Nco I and Xho I restriction enzymes followed by electrophoresis on $1 \%$ agarose gels migrated to the expected size of $\sim 200 \mathrm{bp}$ (Fig. 5).

\section{RNAi of $H$. contortus astacin 3'or 5'end transcripts}

To confirm the absence or presence of transcripts following RNAi treatment, RTPCRs using gene specific primers was carried out in dsRNA treated as well as control worms.

The loss of $H$. contortus astacin 3'or 5'ends cDNA from RNAi-treated worms is shown (Fig. 6), Lane 1: worms soaked with $H$. contortus astacin 3'ends dsRNA; Lane 2: worms soaked with $H$. contortus astacin 5 'end $s$ dsRNA. The transcripts are noticeably visible in the negative control worms, using appropriate primers to check uniformity of the RNA purifications, see lanes $3 \sim 6$, Fig. 6 .

In this paper we describe interference in the expression of astacin-like MTP gene of $H$. contortus in vitro. The RNAi experiments were set up to knock down each $H$. contortus astacin 3'or 5'end $s$ fragment individually. The effects on transcripts and specificity of the silencing following RNAi treatment was demonstrated by RT-PCR's using genespecific primers.

The expression for both gene fragments were non-existent compared to the negative control samples. The RT-PCR results also showed the new dsRNA samples were gene-specific.

However no unusual phenotypes were associated with the F1 and F2 generations, feeding and movement appearing normal in both cases after RNAi. Lack of abnormal phenotypes observed in this study are in agreement with findings elsewhere (Geldhof et al., 2006), who reported that worms treated with individual dsRNA or mixed dsRNA samples had no atypical phenotypes as judged by egg production and viability. Majority of astacin-like MTP genes belong to multicopy of gene families which are highly prevalent in nematode parasites.

Genes within such large families probably show high levels of functional redundancy (Kamath et al., 2003; Geldolf et al., 2005; Geldhof et al., 2006). Compensation for lost function between and within $H$. contortus astacin gene family or its homologues in the parasite could explain why we did not detect abnormal phenotypes.

RNAi offers a powerful tool for gene discovery and functional validation for the vast amount of information available within EST datasets. Traditionally, identification of novel candidate antigens from this big pool of genes is complex, time-consuming and expensive. Consider search for possible vaccine candidates, one of the important questions in the screening process is gene identification, sequence analysis and whether an antigen is accessible by an immune response. 
\begin{tabular}{|l|} 
HCF1NCO- I \\
atgaggctcactatactactgCTCATCTTG GCTGTATCAG CACAGGCAGG CTTATITGAAAAAG CAAAAG GCTIITCAA
\end{tabular}

AGGAGGCAATCTGATTGATAGGATCAAAAACGCGACCCTCACTAGATITGGAAAGATCTITGTGAAGACTGGACT

$\longleftarrow$ HCR1Xho- I
TIITTCATTCggcagcaagttgaaccagatCAGGAAAAAGACGATGAATAAACTGAAACTTACATGGCAGAAGAAGAAG

GTGGAACTGGAAAGCAAGATGAAGGAAATCCTAGCGAGAAGAGACAATACCATTGAGGACTTGAAAGACACGAT

AGTCGAGATCAACGCAGCCAGTAACATCGGCAAACACCTCTTCCAGAGTGATATCCTCTTGACAAAGAAACAAGC

AGATGAAGTCCTGGAAGCTGTCGACGGAACCAGTGGTAGGAAGAAGAGACAAGCGTTCAAAGATAAAAATTATC

CCAATACCACATGGCTAGGAGCACAAGTCTITTACAAGTTTGACGACAGCGCAGACCACTTCACCAGAGAAATGTT

CAAGAAGGGAG CCAAACAATGGGAAGACGTGTCGTGCATCAAGTTCCACCATGATAAGGAGAACAAATCTGAAC

ACAGTATCGTGTTAATCAAAGAGGAAGGCTGTTGGTCCTATGTGGGACGCCTTGGAGGCGAACAACCTCTITCGC TCGGAGTTGGCTGTGAGGAGGTCGGAACTGCGGCACACGaactcggccacgctcttggTCTITCCATACAATGTCTCGT

TACGATCGAGATGACTTCATAACTATCGCTITGGAGAATGTTCGAGAAGATTCGTGGACCAGTACATCAAGGAG ACCACTGAAACTACTACCAACTATGACCTTACCTATGACTACGCCAGCATCATGCATTACGGTGCTACGggtgcttctca

taacaagaaaCCTACAATGATAGCTAACGACGTAAACTTCCAAGAATCGATGGGCTCGCACATCCTGACATTCATAGA

CAAATCCATGATCAACGACCATTATAACTGTAAAGCCAAATGTCTGAATGCCAAGTCTCACCAGTGCAAGAATGGT

GGATTCCCTCACCCAGAAAAATGCTCGGAATGTATITGTCCAAGCGGCTATGGCGGTGCCTTCTGCAATGAACGAC

CTTCTGGATGCGGTAGGAAGTTAGTGGCGAAACAGAGCAAGCAGTTCCTCATTGACAAACTCGGCTTCGGTGGTC

CTGTCAGGGATGAATTCACTITCTGCAACTACTGGATTGAAGCTCCTGAAGGGAAAACGATAGAAGTTAAGATC

Fig.1 H. contortus astacin-like MTPs gene (Acc. no. AM159505)

The forward and reverse primers designated HCF1Nco- I (5'-ccatggatgaggctcactatactactg-3'); HCR1Xho- I (5'ctcgagatctggttcaacttgctgcc-3'); HCF2Nco- I (5'-ccatggaactcggccacgctcttgg-3'); HCR2Xho- I (5'ctcgagttcttgttatgagaagcacc $-3^{\prime}$ ) respectively, are highlighted in the open reading frame. 


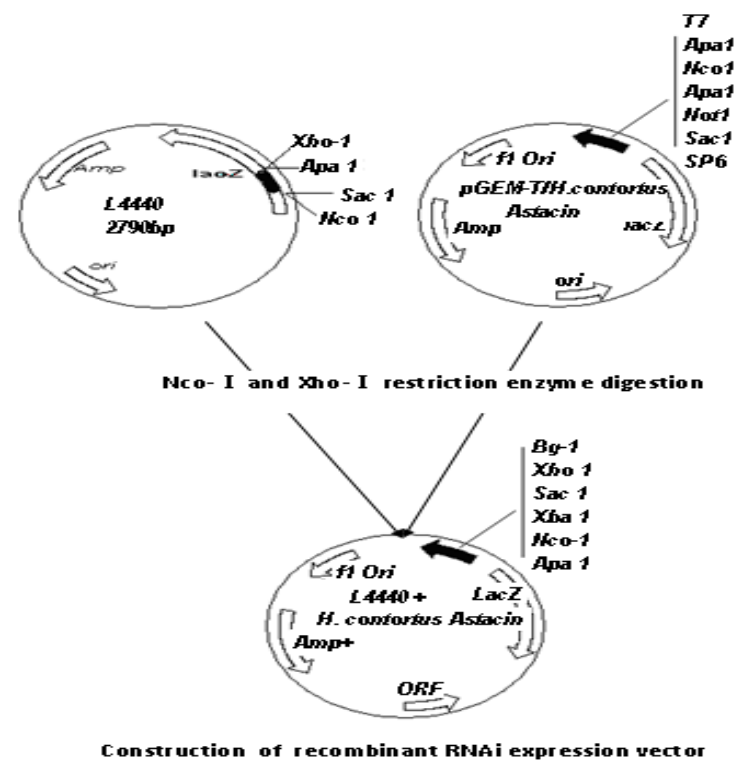

Fig.2 Construction of recombinant L4440/H. contortus astacin expression plasmid

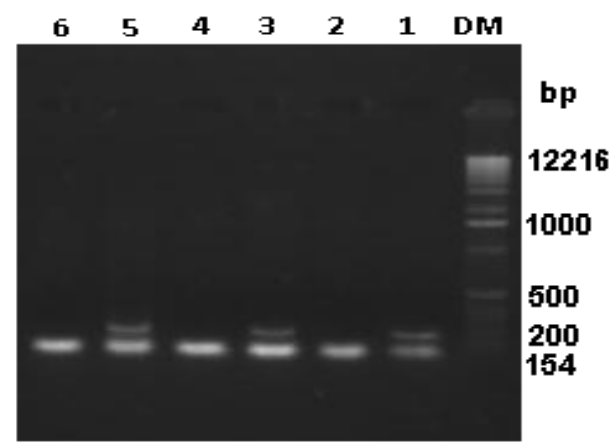

Fig.2 H. contortus astacin 3'or 5'ends PCR products

Lane DM: DNA molecular weight marker $(0.075-12.21 \mathrm{kbp})$. H. contortus astacin amplified in Lane 1: $H$. contortus astacin 3'end $s$ with HCF1=HCR1 primers Lane 3: H. contortus astacin 3'ends with HCF1Nco- I = HCR1Xho- I primers Lane 5: H. contortus astacin 5'ends with HCF2Nco- I = HCR2Xho- I primers. The negative controls in lanes 2, 4 and 6 with no template. Lanes 1, 3 and 5 are PCR products of $\sim 200 \mathrm{bp}$.

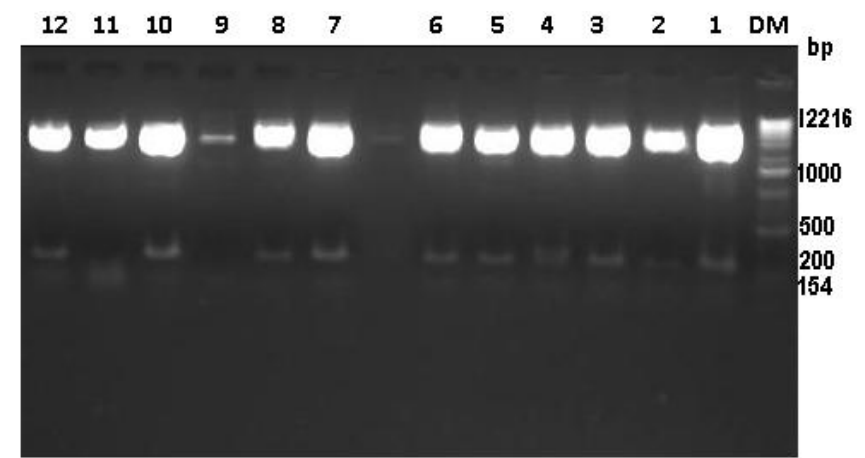

Fig. 3 Plasmids containing inserts of recombinant pGEM -T/ H. contortus astacin 3'or 5'ends Key: DM: DNA molecular weight marker X (0075 - 12 21kbp). Lanes 1 6: recombinant pGEM -T/ H. contortus astacin 3'ends plasmids digested with Nco- I and Xho I enzymes. Lanes 7-12: recombinant pGEM -T/ H. contortus astacin 5'ends plasmids digested with Nco- I and Xho I enzymes. Digested plasmid DNA products were $\sim 200 \mathrm{bp}$ 


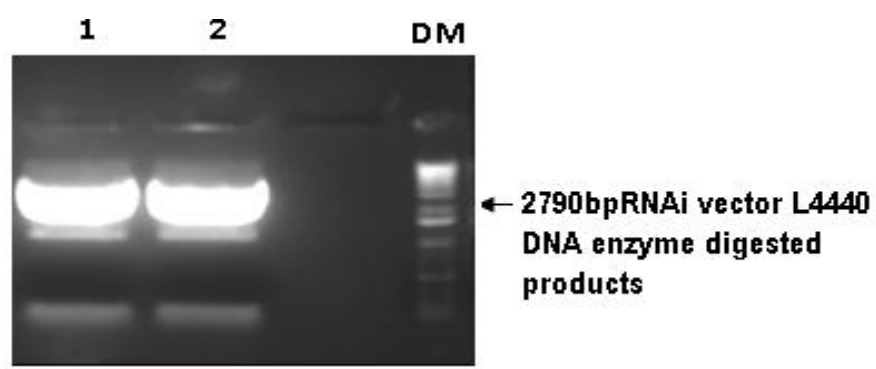

Fig.4 The plasmid of L4440 RNAi vector digested with Nco- I and Xho I restriction enzymes Lane DM: DNA molecular weight marker X (0075 - 12 21kbp); Lanes 1 and 2: Digested L4440 RNAi vector DNA of $\sim 2790 \mathrm{bp}$

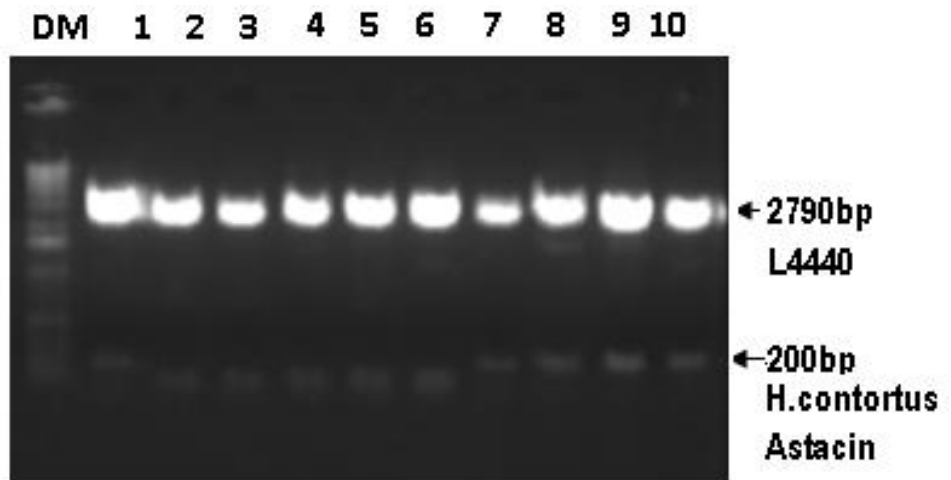

Fig.5 Identification of recombinant plasmids containing inserts of recombinant L4440/ $\mathrm{H}$. contortus astacin 3'or 5'ends DNA plasmids digested with Nco- I and Xho I enzymes

Key: DM: DNA molecular weight marker X (0075 - 12 21kbp). Lanes 1 5: recombinant L4440/ H. contortus astacin 3'ends DNA plasmid digests. Lanes 6-10: recombinant L4440/ H. contortus astacin 5'ends DNA plasmid digests. Digested products were $\sim 200 \mathrm{bp}$.

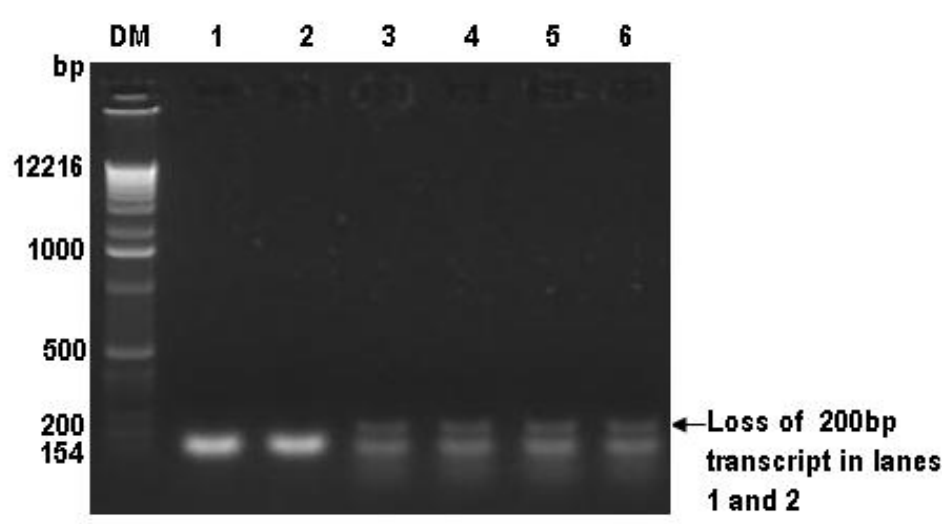

Fig.6 Loss of $H$. contortus astacin 3'or 5'ends transcripts following RNAi. RT-PCRs were carried out using total RNA isolated from RNAi-treated and untreated worms

Key: DM: DNA molecular weight marker X (0075 - 12 21kbp); Lane 1: worms soaked with $H$. contortus astacin 3 'ends dsRNA; Lane 2: worms soaked with $H$. contortus astacin 5'ends dsRNA, lanes $3 \sim 6$ : negative control worms using appropriate primers to check uniformity of the RNA purifications. The major band at $200 \mathrm{bp}$ is lacking from dsRNA treated worms in lanes 1 and 2 for both $H$. contortus astacin 3'or 5'ends transcripts 
The later can only be addressed through immune-localisation studies. It is apparent starting from an expressed sequence tag (EST) it takes months to make a recombinant antigen, raise antiserum, and localise the native protein on worm sections, perform invitro functional tests and run animal vaccination trials. RNAi steps are shorter, specific and could provide the much needed option to the afore-going drawbacks.

\section{Acknowledgements}

This work was supported under the commonwealth scholarship commission academic fellowship in the United Kingdom, ACU, 20-24 Woburn House, Tavistock Square, London, WC1H 9HF. The authors acknowledge generous support given by the entire parasitology lab team at Moredun Research Institute, U.K. (David Smith; George Newlands; Philip Skuce; Alasdair Nisbet; Aileen Halliday; Alison Dicker; Jacqui Matthews; Mairi Mitchell; Rachael Smith; Samantha Ellis; Stephen Smith; Stuart Smith; Frank Turnbull; Keith Ballingall John Parkinson, Alasdair Anthony, James Wasmuth and Ralf Schmid)

\section{References}

Gamble H R, Purcell J P, Fetterer RH (1989). Purification of a $44 \mathrm{kDa}$ protease which mediates the ecdysis of infective Haemonchus contortus larvae. Molecular and Biochemical Parasitology 33, 49-58.

Geldhof P Molloy C, Knox DP (2006). Combinatorial RNAi on intestinal cathepsin B-like proteinases in Caenorhabditis elegans questions the perception of their role in nematode biology. Molecular \& Biochemical Parasitology 145, 128-132.

Geldhof P, Whitton C, Gregory WF, Blaxter M, Knox DP (2005). Characterisation of the two most abundant genes in the Haemonchus contortus expressed sequence tag dataset. International Journal for Parasitology 35, 513-522.

Gomez Gallego S, Loukas A, Slade R W, Neva FA, Varatharajalu R, Nutman TB, Brindley, P J (2005). Identification of an astacin-like metallo-proteinase transcript from the infective larvae of Strongyloides stercoralis. Parasitology International 54, 123-133.

Hashmi S, Britton, C, Liu J, Guiliano DB, Oksov Y, Lustigman S, (2002). Cathepsin $\mathrm{L}$ is essential for embryogenesis and development of Caenorhabditis elegans. J. Biol. Chem. 277, 3477-3486.

Kamath RS, Fraser AG, Dong Y (2003). Systematic functional analysis of the Caenorhabditis elegans genome using RNAi. Nature; 421:220-215.

Loukas A, Bethony JM, Williamson AL, (2004). Vaccination of dogs with a recombinant cysteine protease from the intestine of canine hookworms diminishes the fecundity and growth of worms. J Infect Dis. 1952-61.

Redmond DL and Knox DP. (2004). Protection studies in sheep using affinity purified and recombinant cysteine proteinases of adult Haemonchus contortus. Vaccine, 22:4252-61.

Williamson AL, Brindley PJ, Knox DP, Hotez PJ, Loukas A. (2003). Digestive proteases of blood-feeding nematodes. Trends Parasitol 19, 417-23.

Zhan, B, Hotez PJ, Wang Y, Hawdon JM (2002). A developmentally regulated metalloprotease secreted by hoststimulated Ancylostoma caninum thirdstage infective larvae is a member of the astacin family of proteases. Molecular and Biochemical Parasitology 120, 291-296. 


\section{How to cite this article:}

Charles Muleke Inyagwa and Dave Knox. 2020. RNA Interference of the Hemonchus Contortus Astacin- like MTP Gene by the Soaking Method. Int.J.Curr.Microbiol.App.Sci. 9(04): 1175-1184. doi: https://doi.org/10.20546/ijcmas.2020.904.139 